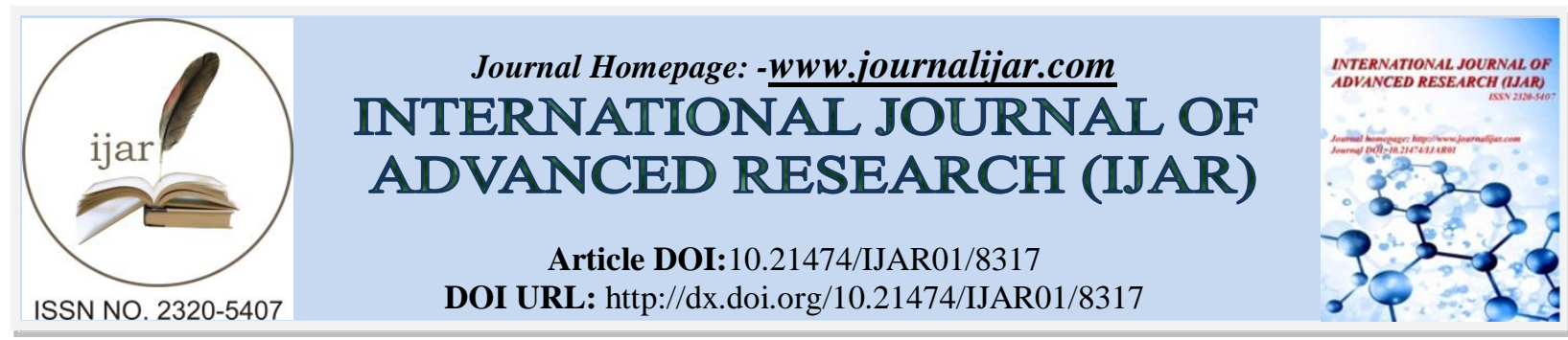

RESEARCH ARTICLE

\title{
PREVALENCE OF PANCREATITIS IN ALCOHOL DEPENDENCE SYNDROME CASES IN A TERTIARY CARE HOSPITAL IN INDIA.
}

1. Assoc Prof(Psy), AFMC Pune, India.

Vinay S Chauhan ${ }^{1}$ and Prateek Yadav ${ }^{2}$.

2. Classified Spl Psychiatry, 151 Base Hospital, India.

\section{Manuscript Info}

Manuscript History

Received: 04 November 2018

Final Accepted: 06 December 2018

Published: January 2019

Keywords:

Pancreatitis; Alcohol; Prevalence ; Nicotine ; MCV.

\section{Abstract}

Background : pancreatitis is a painful, potentially fatal condition. About $1 / 3^{\text {rd }}$ of acute pancreatitis cases in united-states are alcohol induced and $60 \%-90 \%$ have a history of chronic consumption. Many cases of alcoholic pancreatitis do not receive psychiatric intervention for their basic etiology and continue with recurrences. There is further dearth of data in indian context.

Aim : was to find out the prevalence and risk factors for pancreatitis in alcohol dependence syndrome(ads).

Method: a prospective study of newly diagnosed patients of ads, admitted in tertiary care hospital from jan to dec 2016, was carried out. Socio-demographic data, quantity and duration of alcohol consumption, co-morbid nicotine dependence were tabulated. Pancreatitis was diagnosed on basis of icd-10, k-85 criteria.

Results:total of 140 cases of ads were admitted during the period. 26 cases $(18.58 \%)$ were having pancreatitis. Among pancreatitis group, most had 6-10 years(73.1\%) of drinking and were drinking about $80 \mathrm{gm} /$ day $(50 \%)$. $76.9 \%$ among pancreatitis group had nicotine dependence. Serum bilirubin, mcv, ast, alt \& ggt were raised in $23.1 \%, 57.7 \%, 46.2 \%, 42.3 \%$ and $96.2 \%$ respectively. The difference between pancreatitis and non pancreatitis group were statistically significant $(\mathrm{p}=0.001)$ for co-morbid nicotine dependence syndrome and for $\mathrm{mcv}(\mathrm{p}=0.26)$. Rest variables did not show significance. Important finding was that $18.58 \%$ of ads patients were having pancreatitis. Conclusion:timely intervention in these could prevent future recurrences and thus it is imperative that attention to the root cause of the condition, alcohol is paid rather than just responding to its effects.

Copy Right, IJAR, 2018,. All rights reserved.

\section{Introduction:-}

The World Health Organization (WHO) estimates that there are about 2 billion people worldwide who consume alcoholic beverages and 76.3 million with diagnosable alcohol use disorders.(1) The 2010 analysis of 67 risk factors and risk factor clusters for death and disability found that alcohol consumption was the third leading risk factor for death and disability accounting for 5.5\% of disability-adjusted life years (DALYs) lost globally.(2) Overall, there is a causal relationship between alcohol consumption and more than sixty types of disease and injury. In India, alcohol consumption is one among the top ten risk factors and attributable to nearly $3 \%$ of DALYs lost.(3) One of the 
important digestive organs adversely affected by alcohol abuse is the pancreas which was recognized as early as 1878 by Freidreich who wrote 'I am inclined to believe that a general chronic interstitial pancreatitis may result from excessive alcoholism (drunkard's pancreas)'.(4) While Freidreich's observation referred to the association with chronic pancreatic injury, it is now widely acknowledged that the clinical spectrum of alcohol induced pancreatic injury ranges from isolated acute episodes to repeated acute attacks and finally to chronic manifestations. Alcohol induced pancreatitis, is potentially fatal and the course may be short with acute manifestations or long term (chronic). $(5,6)$

Alcohol as an etiology has not been quantified however experts define consumption of over 50-80g (4-7 drinks/day) with or without a minimum drinking duration (7,8). An international consensus defined alcoholic chronic pancreatitis based on typical clinical history, threshold alcohol consumption $(80 \mathrm{~g}$ or more of alcohol for a few years in males and less in females) and morphological evidence of chronic pancreatitis on imaging studies or histology(9). A meta-analysis found a monotonic and approximately exponential dose-response relationship between average volume of alcohol consumption and pancreatitis (10). Another meta-analysis analyzed all studies published between 1980 and 2008 and concluded that the threshold between alcohol consumption and pancreatitis is 4 drinks daily (11). About $70 \%$ of pancreatitis cases are believed to be attributable to chronic, heavy alcohol consumption but this percentage differs between countries (12).

Prolonged consumption for 5-10 years typically precedes the initial attack and risk increases with the amount, which suggests a direct toxic effect of alcohol. By causing oxidative stress in the gland; and promoting the synthesis of pancreatic digestive enzymes and destabilizing intracellular membranes, the gland is predisposed toautodigestion. $(13,14,15,16)$

Major observation that has driven research is that only few alcoholics develop pancreatitis. This suggests the presence of specific susceptibility or trigger factors. However, no single, clear-cut susceptibility factor has yet been identified.(17) The role of smoking is controversial, although researchers agree that this is a major cause through the development of aero-digestive tract cancers and cardiovascular disease.(18) There are hardly any Indian studies on ascertaining the prevalence of Pancreatitis in ADS cases and probable risk factors in these patients. Hence the present study was conducted with the aim to find out the prevalence and risk factors for pancreatitis in ADS and to quantify alcohol as causal factor.

\section{Materials and Methods:-}

This was a prospective study carried out in a tertiary care hospital. All patients referred for alcohol use disorder during Jan 2016 to Dec 2016 were studied. They were diagnosed with ADS or Nicotine Dependence Syndrome as per ICD-10-DCR criteria. The relevant information was tabulated. Pancreatitis was diagnosed on the basis of ICD10, K-85 criteria. Serum bilirubin, MCV, AST, ALT and GGT were considered to be raised for value more than 1.0 $\mathrm{mg} / \mathrm{dl}, 96 \mathrm{fl}, 45 \mathrm{IU} / \mathrm{L}, 45 \mathrm{IU} / \mathrm{L}$ and $85 \mathrm{IU} / \mathrm{L}$ respectively.

Acute Pancreatitis(ICD-10-K-85) Diagnosis requires 2 of the 3 following criteria:Characteristic acute onset of epigastric or vague abdominal pain that may radiate to the back, Serum amylase or lipase levels $\geq 3$ times the upper limit of normal and imaging study with characteristic changes. CT, MRI, abdominal ultrasound or endoscopic ultrasound can be used for diagnosis

Since the main aim was to find out prevalence and risk factors for alcoholic pancreatitis; patients having pancreatitis with ADS were studied in detail. All subject participants were male patients as the hospital caters largely for male population. Statistical analysis was done using SPSS version 21.

\section{Results:-}

A total of 140 cases were diagnosed as cases of ADS during Jan 2016 to Dec 2016. The mean age was 36.13 years. Table 1 depicts demographic characteristics of the study participants. $18.5 \%$ of ADS cases were found to have acute pancreatitis during the study period. Total of 34 male cases of acute Pancreatitis were admitted in this hospital during above period. $26(76 \%)$ cases were found to have ADS (included in the 140 cases of ADS of study group) and $08(24 \%)$ were diagnosed to be idiopathic pancreatitis. 09 patients came under psychiatric attention on their second admission as relapse of acute pancreatitis. 
Distribution of ADS patients who suffered from Acute Pancreatitis as per age group, monthly income, education and occupation depicted in Table-2. The maximum number of ADS patients who suffered from Acute Pancreatitis was in age group 30-39 years (65.4\%), income group 20,000-29,999 (69\%), education up to high school (50\%) and semiskilled \& unskilled workers (73\%). However, none of the variables (age, income, education and occupation) showed statistically significant linear trend $(\mathrm{p}=0.66,0.17,0.73$ and 0.81 respectively).

Table 3 depicts various parameters studied between Pancreatitis and Non pancreatitis group among the study participants of ADS patients. The mean duration of drinking was 9.77years and mean quantity of drinking was 83 gms/day considering 140 study participants. The maximum number among pancreatitis group had 6-10 years (73.1\%) of drinking and were drinking about $80 \mathrm{gms} /$ day(50\%). 76.9\% among pancreatitis group had nicotine dependence syndrome. The difference between Pancreatitis and Non pancreatitis group were statistically significant ( $\mathrm{p}=0.001$ ) for co-morbid nicotine dependence syndrome and for MCV ( $\mathrm{p}=0.026)$. Rest of the variables did not show statistically significant difference between Pancreatitis and Non Pancreatitis group.

Table1:-Demographic characteristics of study participants

\begin{tabular}{|lll|}
\hline Characteristic & N & $\%$ \\
\hline Age group(years) & & 14.3 \\
\hline $20-29$ & 20 & 60.7 \\
\hline $30-39$ & 85 & 20.7 \\
\hline $40-49$ & 29 & 4.3 \\
\hline$>49$ & 06 & \\
\hline Monthly Income & & 6.4 \\
\hline$<20,000$ & 09 & 30.7 \\
\hline $20,000-24,999$ & 43 & 42.9 \\
\hline $25,000-29,999$ & 60 & 20.0 \\
\hline$\geq 30,000$ & 28 & \\
\hline Education & & 44.3 \\
\hline Up to high school & 62 & 40.0 \\
\hline Intermediate & 56 & 15.7 \\
\hline Graduate & 22 & \\
\hline Occupation & & 26.4 \\
\hline Skilled & 37 & 43.6 \\
\hline Semiskilled & 61 & 30.0 \\
\hline Unskilled & 42 & \\
\hline
\end{tabular}

Table 2:-Distribution of Alcohol dependence syndrome patients who suffered from Acute Pancreatitis as per age group, monthly income, education and occupation

\begin{tabular}{|lccc|}
\hline Characteristic & $\mathrm{N}$ & $\%$ & $\mathrm{p}$ value \\
\hline Age group (years) & & 11.5 & 0.662 \\
\hline $20-29$ & 03 & 65.4 & \\
\hline $30-39$ & 17 & 7.7 & \\
\hline $40-49$ & 04 & & \\
\hline$>49$ & 02 & & 0.172 \\
\hline & & 15.4 & \\
\hline Monthly Income & & 34.6 & \\
\hline$<20,000$ & 04 & 34.6 & 0.737 \\
\hline $20,000-24,999$ & 09 & 15.4 & \\
\hline $25,000-29,999$ & 09 & & \\
\hline$\geq 30,000$ & 04 & 30.0 & \\
\hline Education & & 11.5 & \\
\hline Up to high school & 13 & & \\
\hline Intermediate & 10 & & \\
\hline Graduate & 03 & & \\
\hline Occupation & & & \\
\hline
\end{tabular}




\begin{tabular}{|llll|}
\hline Skilled & 07 & 26.4 & 0.810 \\
\hline Semiskilled & 10 & 38.4 & \\
\hline Unskilled & 09 & 34.6 & \\
\hline
\end{tabular}

Table 3:-Association between Pancreatitis and Non Pancreatitis group of Alcohol dependence syndrome patients

\begin{tabular}{|c|c|c|c|}
\hline Characteristic & Pancreatitis & Non-Pancreatitis & $\mathrm{p}$ value \\
\hline \multicolumn{4}{|c|}{ Duration of drinking (years) } \\
\hline $0-5$ & 01 & 16 & 0.152 \\
\hline $6-10$ & 19 & 62 & \\
\hline $11-15$ & 04 & 32 & \\
\hline$>15$ & 02 & 04 & \\
\hline \multicolumn{4}{|c|}{ Quantity of drinking (Gms) } \\
\hline $60 \mathrm{gms}$ & 04 & 32 & 0.477 \\
\hline $80 \mathrm{gms}$ & 13 & 45 & \\
\hline $100 \mathrm{gms}$ & 06 & 29 & \\
\hline $120 \mathrm{gms}$ & 03 & 08 & \\
\hline \multicolumn{4}{|c|}{ Nicotine dependence syndrome } \\
\hline Yes & 20 & 46 & $0.001 * *$ \\
\hline No & 06 & 68 & \\
\hline \multicolumn{4}{|l|}{ MCV } \\
\hline Raised & 15 & 39 & $0.026^{*}$ \\
\hline Not Raised & 11 & 75 & \\
\hline \multicolumn{4}{|c|}{ Serum Bilrubin } \\
\hline Raised & 06 & 18 & 0.373 \\
\hline Not Raised & 20 & 96 & \\
\hline \multicolumn{4}{|l|}{ AST } \\
\hline Raised & 12 & 61 & 0.498 \\
\hline Not Raised & 14 & 53 & \\
\hline \multicolumn{4}{|l|}{ ALT } \\
\hline Raised & 11 & 67 & 0.127 \\
\hline Not Raised & 15 & 47 & \\
\hline \multicolumn{4}{|l|}{ GGT } \\
\hline Raised & 25 & 108 & 0.764 \\
\hline Not Raised & 01 & 06 & \\
\hline
\end{tabular}

\section{Discussion:-}

Excessive consumption of alcohol is a major cause of acute and chronic pancreatitis in both developed and developing countries. Alcohol has been found as the causative factor of acute mild pancreatitis and severe acute pancreatitis in $41 \%$ and $18 \%$ respectively, which was more than Gall stones associated pancreatitis.(19) Our study revealed $76 \%$ of acute pancreatitis cases were alcohol related, other studies have findings with rates ranging from $70 \%-89 \% .(5,20)$

Twenty six (18.5\%) patients suffered from acute pancreatitis among 140 cases of ADS in the study. This finding has not been replicated probably as most of the studies are carried out by gastroenterologist and mostly in chronic pancreatitis group. This is a highlight of the study particularly in the Indian context and brings forth the high number of people in this group who suffer from this near fatal disorder, in a scenario when more than two-third of the sample either had less than ten yrs of consumption or less than $80 \mathrm{gm}$ of consumption each day(mean-9.77 years and mean quantity - 83 gms/day)

Maximum prevalence were among 30-39 yrs age group(65\%), who were drinking about 80 gms/day(50\%) for 6-10 years(73\%), other studies have also documented about same $(5,12)$. This shows that a prolonged exposure is required in majority, for development of pancreatitis, and also probably hints that excessive consumption is not the 
only reason as people with more amount and years of drinking than the above reported lesser fraction in the pancreatitis group, although substantiating it is beyond the scope here.

As the results show, the proportion of patients detected to have Pancreatitis increases exponentially if the duration of drinking is more than 5yrs (from 1/16 to almost 1/5) although after that further duration has little impact. Similarly the amount of alcohol consumed has exponential effects if it is more than 60gms per day. (table3).

Smoking also confers a strong, independent and dose-dependent risk of pancreatitis that may be additive or multiplicative when combined with alcohol.(23) Co-morbid Nicotine dependence syndrome was seen in $76.9 \%$ of the pancreatitis group and was highly statistically significant, which has been a finding in other studies $(21,22)$.The mechanism by which smoking contributes to pancreatic injury or by which smoking accelerates the pancreatic inflammatory process is still unknown but laboratory studies have found that activation of multiple signal transduction pathways due to nicotine exposure results in high levels of intracellular calcium release and may be responsible for cell cytotoxicity and cell injury.(23)

Other various alcohol related lab parameters were studied for both groups among cases of ADS; the significant statistical association was found with MCV. The MCV has been found to be independently associated with higher alcohol consumption and alcohol related diseases such as esophageal carcinoma, chronic alcoholic pancreatitis, and liver cirrhosis. (24) Thus a higher MCV value is associated with Pancreatitis as it indicates higher alcohol consumption over a prolonged period of time, both of which are indicated in the precipitation of the disease.

The strengths of the study were clinical relevance of pancreatitis association with ADS \& portrayal of its prevalence in pancreatitis, sample size, accurate diagnostic criteria and. Further the study also included Acute Pancreatitis patients, which has limited research. The study was limited with all patients being males and absence of control.

The morbidity and mortality of Acute Pancreatitis and the propensity to progress to a chronic course warrant that a preventive strategy is the best management for this disease, however the management of alcoholic pancreatitis is mostly reactive; little is done to prevent disease progression. Measures to reduce alcohol consumption are not even mentioned in many published guidelines for management of alcoholic pancreatitis. There is ample evidence available that abstinence decreases the frequency and severity of attack.(14) It has been found that, at least in the short to medium term, specific interventions and/or counseling measures to reduce alcohol consumption result in a demonstrable reduction in attacks of alcoholic pancreatitis.(25)

\section{Conclusion:-}

Therapeutic imperative is that the routine approach to alcoholic pancreatitis is expanded to include measures to emphasize the importance of abstinence and to support the patient in their efforts to minimize their drinking. As such, we envisage that these interventions will be performed not only by the specialist, but perhaps more importantly, by general practitioners, nursing or allied health staff who would be likely to see the patient on a regular basis

\section{References:-}

1. World Health Organization. Global Status Report on Alcohol and Health 2014. Geneva, Switzerland: World HealthOrganization;2014.

2. Lim SS et al. A comparative risk assessment of burden of disease and injury attributable to 67 risk factors and risk factor clusters in 21 regions, 1990-2010: A systematic analysis for the Global Burden of Disease Study 2010. Lancet $2012 ; 380: 2224-60$.

3. Ray R et al. The Extent, Pattern and Trends of Drug Abuse in India: National Survey. Government of India, New Delhi, India: United Nations Office on Drugs and Crimes and Ministry of Social Justice and Empowerment; 2004.

4. Friedreich N. Cyclopedia of the Practice of Medicine. New York: William Wood; 1878. Disease of the pancreas.

5. Marta Herreros-Villanueva etal. Alcohol consumption on pancreatic diseases. World J Gastroenterol. 2013 Feb 7; 19(5): 638-647

6. Layer $\mathrm{P}$ etalThe different courses of early- and late-onset idiopathic and alcoholic chronic pancreatitis. Gastroenterology. 1994;107:1481-1487. 
7. Ammann RW, Muellhaupt B. Progression of alcoholic acute to chronic pancreatitis. Gut. 1994;35:552-556.

8. Ammann RW. A clinically based classification system for alcoholic chronic pancreatitis: summary of an international workshop on chronic pancreatitis. Pancreas. 1997;14:215-221.

9. Harper SJ, Cheslyn-Curtis S. Acute pancreatitis. Ann ClinBiochem. 2011;48:23-37.

10. Corrao G, Bagnardi V, Zambon A, La Vecchia C. A meta-analysis of alcohol consumption and the risk of 15 diseases. Prev Med. 2004;38:613-619.

11. Irving HM, Samokhvalov AV, Rehm J. Alcohol as a risk factor for pancreatitis. A systematic review and metaanalysis. J Pancreas. 2009;10:387-392.

12. Dufour MC, Adamson MD. The epidemiology of alcohol-induced pancreatitis. Pancreas. 2003;27:286-290.

13. Yadav D, Lowenfels AB. Trends in the epidemiology of the first attack of acute pancreatitis: a systematic review. Pancreas. 2006;33:323-330.

14. Minoti V. Apte; Romano C. Pirola; Jeremy S. Wilson. Pancreas: Alcoholic Pancreatitis-It's the Alcohol, Stupid. Nat Rev GastroenterolHepatol. 2009;6(6):321-322

15. Minoti V. Apte; Romano C. Pirola; Jeremy S. Wilson. Mechanism of Alcoholic pancreatitis. Journal of Gastroenterology and Hepatology. 2010;(25):1816-1826

16. Witt, H., Apte, M. V., Keim, V. \& Wilson, J. S. Chronic pancreatitis: challenges and advances in pathogenesis, genetics, diagnosis, and therapy. Gastroenterology 132, 1557-1573 (2007).

17. Apte, M. V. \& Wilson, J. S. Mechanisms of pancreatic fibrosis. Dig. Dis. 22, 273-279 (2004).

18. Apte, M. V., Pirola, R. C. \& Wilson, J. S. Where there's smoke there's not necessarily fire. Gut 54, 446-447 (2005).

19. Baig SJ, Rahed A, Sen S. A prospective study of the aetiology, severity and outcome of acute pancreatitis in Eastern India.TropGastroenterol. 2008 Jan-Mar;29(1):20-2.

20. Avadhani KG, Shirshetty AK. An epidemiological study of acute pancreatitis in rural population. J Med Sci Health 2016;2(3):25-29.

21. Yadav d etal. Alcohol consumption, cigarette smoking, and the risk of recurrent acute and chronic pancreatitis. Arch intern med. 2009 jun 8;169(11):1035-45

22. DhirajYadav\& David C. Whitcomb The role of alcohol and smoking in pancreatitisNature Reviews Gastroenterology and Hepatology 7, 131-145 (March 2010)

23. Chowdhury P, MacLeod S, Udupa KB, et al. Pathophysiological effects of nicotine on the pancreas: An update. ExpBiol Med 2002;227:445-54.

24. Leimin Sun etal. Mean Corpuscular Volume and ADH1C Genotype in White Patients With Alcohol-Associated Diseases.Alcoholism. 2005( 29), 5,788-793.

25. Nordback, I. et al. The recurrence of acute alcohol-associated pancreatitis can be reduced: a randomized controlled trial. Gastroenterology 136, 848-855 (2009). 